Revista Brasileira de Agricultura Irrigada v.10, nº.4, p. 858 - 865, 2016

ISSN 1982-7679 (On-line)

Fortaleza, CE, INOVAGRI - http://www.inovagri.org.br

DOI: $10.7127 /$ rbai.v10n400461

Protocolo 461.16 - 18/07/2016 Aprovado em 10/08/2016

\title{
O EFEITO DAS MUDANÇAS CLIMÁTICAS NAS NECESSIDADES HÍDRICAS DA MELANCIEIRA, NO PIAUÍ UTILIZANDO O “CROPWAT”
}

\author{
Kleiton Rocha Saraiva ${ }^{1}$, Carlos Diniz Félix Junior ${ }^{2}$, Rodrigo Moura Ibiapina ${ }^{3}$, Henrique César \\ Sena Gomes ${ }^{4}$, Guilherme Ibiapina Reinaldo ${ }^{5}$
}

\section{RESUMO}

Objetivou - se quantificar o impacto do aumento da temperatura previsto pelo Intergovernamental Panel on Climate Change - IPCC, na demanda hídrica da cultura da melancieira, para os cenários B2 (previsão de aumento da temperatura anual de até $3,8^{\circ} \mathrm{C}$ ) e A2 (previsão de aumento da temperatura anual de até $5,4^{\circ} \mathrm{C}$ ), comparando com a demanda hídrica da cultura em condições climáticas atuais de Teresina - PI. Para determinar as necessidades de água da cultura foi utilizado o modelo "CROPWAT”. Este calcula a evapotranspiração de referência (ETo) e a evapotranspiração das culturas (ETc), além de realizar o balanço hídrico no solo. Na determinação da ETc foram utilizados dados climáticos de Teresina, PI, levando-se em consideração o aumento da temperatura, de acordo com previsões do IPCC, e coeficientes da cultura $(\mathrm{Kc})$ da melancieira, obtidos através de experimentos locais. A pesquisa comprovou que com o aumento da temperatura, previsto pelo IPCC para as próximas décadas, a demanda de água da cultura da melancieira deverá ser, em termos médios, de 5,9\% (cenário B2) a 11,9\% (cenário A2) maior, para as condições climáticas de Teresina, PI.

Palavras-chave: água, software, evapotranspiração.

\footnotetext{
${ }^{1}$ Instituto Federal do Piauí - IFPI, Docente. Campo Maior, Piauí, Brasil. kleiton.rocha@ifpi.edu.br. (86) 994776236. Av. Nilo de Oliveira Santana, S/N, localidade Fazendinha, Campo Maior, PI, CEP: 64.280-000.

${ }^{2}$ Instituto Federal do Piauí - IFPI, Aluno do Curso Técnico em Agricultura. Campo Maior, Piauí, Brasil. junior.diniz.felix@hotmail.com. (86) 994631505. Av. Nilo de Oliveira Santana, S/N, localidade Fazendinha, Campo Maior, PI, CEP: 64.280-000.

${ }^{3}$ Instituto Federal do Piauí - IFPI, Aluno do Curso Técnico em Agricultura. Campo Maior, Piauí, Brasil. rodrigoibi4123@gmail.com. (86) 994132437. Av. Nilo de Oliveira Santana, S/N, localidade Fazendinha, Campo Maior, PI, CEP: 64.280-000.

${ }^{4}$ Instituto Federal do Piauí - IFPI, Aluno do Curso Técnico em Agricultura. Campo Maior, Piauí, Brasil. henriquecesar321@hotmail.com. (86) 981737760. Av. Nilo de Oliveira Santana, S/N, localidade Fazendinha, Campo Maior, PI, CEP: 64.280-000.

${ }^{5}$ Instituto Federal do Piauí - IFPI, Aluno do Curso Técnico em Agricultura. Campo Maior, Piauí, Brasil. guiibiapina@gmail.com. (86) 994132437. Av. Nilo de Oliveira Santana, S/N, localidade Fazendinha, Campo Maior, PI, CEP: 64.280-000.
} 


\title{
THE EFFECT OF CLIMATE CHANGE ON WATER NEEDS OF WATERMELON IN PIAUI USING THE "CROPWAT"
}

\begin{abstract}
If aimed to quantify the impact of rising temperatures predicted by the Intergovernamental Panel on Climate Change - IPCC, the water demand of the culture of watermelon for the B2 scenarios (increase forecast annual temperature up to $3.8^{\circ} \mathrm{C}$ ) and $\mathrm{A} 2$ (increase forecast annual temperature up to $5.4^{\circ} \mathrm{C}$ ) compared with the crop water demand in the current climate of Teresina - PI. To determine crop water needs model was used "CROPWAT". This calculates the reference evapotranspiration (ETo) and crop evapotranspiration (ETc), and perform the water balance in the soil. In determining the ETc were used weather data from Teresina, PI, taking into account the temperature rise, according to forecasts of the IPCC, and crop coefficients (Kc) of watermelon, obtained through local experiments. Research has shown that with increasing temperature, provided by the IPCC for the coming decades, the demand for water culture of the watermelon should be, on average, $5.9 \%$ (B2 scenario) to $11.9 \%$ (scenario A2 ) increased, for the climatic conditions of Teresina, PI.
\end{abstract}

Keywords: water, software, evapotranspiration.

\section{INTRODUÇÃO}

A agricultura irrigada utiliza entre 70 e $80 \%$ da água doce disponível no mundo (SARAIVA et al., 2013). Segundo Annan (2005) a produção global de alimentos terá que aumentar em $60 \%$, durante o período de 2000 a 2030, para satisfazer a crescente demanda populacional. Isso acarretará num aumento de $14 \%$ na demanda de água para a agricultura irrigada.

Segundo o IBGE (2010), em termos de Brasil, foram produzidas $2.052 .928 \mathrm{t}$ de melancia em uma área plantada de 96.477 ha. Todavia, as regiões Nordeste e Sul são as principais produtoras da hortaliça, com $34,15 \%$ e $24,64 \%$, respectivamente.

A cultura da melancieira, por ser de fácil cultivo, apresentar ciclo curto, e ter boa aceitação no mercado é uma das culturas mais exploradas e consumidas no Nordeste brasileiro, inclusive no Piauí. Na safra 2015/2016 a cultura alcançou os maiores níveis de exportação da última década (SALES et al., 2016).

Assim como para a quase totalidade das culturas, um dos fatores mais importantes no cultivo da melancia é o manejo de irrigação (FIGUEIRÊDO et al., 2009).
No entanto, sabe-se que o consumo de água pelas culturas é diretamente afetado pelas mudanças do clima (SARAIVA; SOUZA, 2013). Segundo a definição do Painel Intergovernamental para as Alterações Climáticas (IPCC), entende-se por alterações climáticas as alterações significativas no estado do clima, que podem ser identificadas por variações de médias e/ou medidas de dispersão de variáveis climáticas, e que persistem por longos períodos, tipicamente décadas ou períodos ainda mais longos (ABREU; PEREIRA, 2010).

No atual contexto das mudanças climáticas, a implementação de medidas efetivas de adaptação regional é tão importante quanto a prevenção do aquecimento global. Por sua vez, essas medidas de adaptação serão tão mais efetivas quanto melhor se conheçam as causas e tipos de vulnerabilidades existentes em cada região, ou mesmo em cada território. As vulnerabilidades encontradas no Semiárido Brasileiro (SAB) são diferentes entre si, assim como são de natureza e consequências distintas das encontradas em outras regiões sujeitas à escassez hídrica (VIANNA et al., 2012).

Nos cem anos que medeiam entre $1905 \mathrm{e}$ 2006, a temperatura do ar sobre a terra aumentou $0,74{ }^{\circ} \mathrm{C}$, enquanto que nos 50 anos 
entre 1956 e 2005 esse aumento foi de $1,30^{\circ} \mathrm{C}$, o que representa quase o dobro do valor anterior (IPCC, 2007). Segundo Marengo (2008), nos últimos 50 anos a temperatura média aumentou $0,7^{\circ} \mathrm{C}$. No Nordeste, nos últimos 40 anos registraram-se aumentos de 1,5 a $2^{\circ} \mathrm{C}$ na temperatura máxima (SANTOS et al. 2010). Segundo Diaz et al. (2008) mesmo com previsão do IPCC para frequentes precipitações em algumas regiões, com 0 aumento da temperatura prevista (previsões do IPCC através dos cenários B2 e A2), a evapotranspiração será maior em todos os meses do ano, aumentando a demanda hídrica das culturas.

O modelo CROPWAT é adotado pela FAO (Food Agriculture Organization) para o cálculo das demandas hídricas das culturas e elaboração de calendários de irrigação. O CROPWAT tem sido utilizado em vários países e aplicações foram realizadas para as condições da agricultura irrigada brasileira (MINUZZI et al., 2014; SALES et al., 2015).

Poucos estudos foram realizados no Brasil, utilizando modelos de simulação, para analisar os impactos das mudanças climáticas na produtividade das culturas (SILVA JÚNIOR et al., 2007). Portanto, a pesquisa objetivou quantificar o impacto do aumento da temperatura previsto pelo IPCC, na demanda hídrica da cultura da melancieira, para os cenários B2 (previsão de aumento da temperatura anual de até $3,8^{\circ} \mathrm{C}$ ) e $\mathrm{A} 2$ (previsão de aumento da temperatura anual de até $5,4^{\circ} \mathrm{C}$ ), comparando com a demanda hídrica da cultura em condições climáticas atuais de Teresina - PI.

\section{MATERIAL E MÉTODOS}

Para determinar as necessidades hídricas da cultura da melancia foi utilizado o "software" CROPWAT, desenvolvido pela FAO (Food Agriculture Organization). Esse "software" foi utilizado para calcular a ETo e a ETc, além de ser capaz de realizar o balanço hídrico do solo. Para determinar a evapotranspiração da cultura (ETc) foram utilizados dados climáticos da cidade de
Teresina - PI, oriundos de estação meteorológica. Já os dados da cultura foram obtidos através de resultados experimentais locais.

Para o cálculo da Evapotranspiração de referência (ETo), o CROPWAT utilizou a equação de Penman-Monteith/FAO, recomendado por Allen et al. (1998). Para tanto, o modelo utilizou as seguintes informações climáticas: (a) temperatura máxima, Tmáx; (b) temperatura mínima, Tmin; (c) umidade relativa do ar, Ur; (d) velocidade do vento, Vv; e, (e) insolação, In. Também foram necessárias a altitude e a latitude do local. O tipo climático da região, de acordo com a classificação de Köppen, é AW. Os dados climatológicos utilizados neste trabalho foram obtidos através de normais climatológicas. Para a utilização no CROPWAT foram calculadas as médias mensais dos parâmetros.

Os dados referentes à cultura foram: (a) duração das fases fenológicas da cultura; (b) coeficiente da cultura (Kc); (c) profundidade das raízes $(\mathrm{Z})$; e, (d) nível de depleção da água do solo (p). A duração de cada fase do ciclo de desenvolvimento da melancia bem como dos valores de Kc utilizados neste trabalho, foram extraídos de Silva et al. (2015); sendo possível a determinação da ETc da melancia.

Com as informações do clima e da cultura da melancia foram calculadas pelo CROPWAT as necessidades de água, de acordo com a época de plantio simulada (01/09/2016). Posteriormente, com a utilização dos mesmos Kc's, mas com variação na ETo, decorrente das mudanças do clima (dados do Painel Intergovernamental para as Alterações Climáticas - IPCC, relacionados ao aumento da temperatura), o CROPWAT foi novamente utilizado (simulações), gerando novas ETc's. A ETc foi calculada para os cenários B2 (otimista), em que o aquecimento a nível anual pode chegar a $3,8^{\circ} \mathrm{C}$ (aumento acrescido à temperatura atual) e para o cenário A2 (pessimista) em que o aquecimento poderá chegar a $5,4^{\circ} \mathrm{C}$ no Brasil (IPCC, 2007).

Finalmente, foram comparadas as necessidades hídricas da cultura da melancia nas condições climáticas atuais, com as 
necessidades hídricas para os dois cenários de aumento da temperatura.

\section{RESULTADOS E DISCUSSÃO}

Na Tabela 1 constam, a cada fase fenológica da cultura: o Kc oriundo de experimentos locais, a ETo calculada pelo CROPWAT, com a utilização de variáveis climáticas atuais e a ETc.

Tabela 1. Variáveis referentes à evapotranspiração da cultura da melancia: ETo (Evapotranspiração de Referência), Kc (Coeficiente de cultivo) e ETc (Evapotranspiração da cultura).

\begin{tabular}{cccc}
\hline Fase fenológica & ETo $\left(\mathrm{mm} \cdot \mathrm{dia}^{-1}\right)$ & Kc & ETc $\left(\mathrm{mm}^{-1 \mathrm{dia}^{-1}}\right)$ \\
\hline Inicial & 4,47 & 0,25 & $1,10-1,22$ \\
Desenvolvimento & $4,47-4,65$ & $0,57-1,36$ & $1,22-6,20$ \\
Intermediaria & 4,65 & 1,36 & $6,20-4,86$ \\
Final & $4,65-4,30$ & 0,61 & $4,86-2,95$ \\
\hline
\end{tabular}

A ETc obtida a partir de dados de Kc's locais e de ETo calculada através de série histórica (média histórica e atuais), na fase inicial do ciclo da cultura da melancia, variou de 1,10 a $1,22 \mathrm{~mm} \mathrm{dia}^{-1}$. Com resultado semelhante, Bastos et al. (2012) analisando ETc e Kc da melancia, no Estado do Piauí, para a mesma fase fenológica, encontraram ETc de $1,18 \mathrm{~mm} \mathrm{dia}^{-1}$.

Já na fase de desenvolvimento (crescimento vegetativo), em que a demanda hídrica da cultura se eleva, a ETc variou de $1,22 \mathrm{~mm} \mathrm{dia}^{-1}$ a $6,20 \mathrm{~mm} \mathrm{dia}{ }^{-1}$. Como verificado nos resultados de Miranda et al. (2004) há um aumento considerável nessa fase fenológica da cultura. Esse aumento pode ser justificado devido ao aumento da área foliar e ao crescimento do sistema radicular da melancia, durante essa fase fenológica. Em condições climáticas semelhantes, Bastos et al. (2012) verificaram, também na fase de desenvolvimento da cultura, ETc variando de 3,8 $\mathrm{mm} \mathrm{dia}^{-1}$ a $8,1 \mathrm{~mm} \mathrm{dia}^{-1}$; já Silva et al. (2015) encontraram ETc mínima de 3,0 mm $\mathrm{dia}^{-1}$ e máxima de $6,8 \mathrm{~mm} \mathrm{dia}^{-1}$. Verifica-se que, ao analisar as médias dos autores, essas são semelhantes à média verificada nesta pesquisa.
Durante a fase final do ciclo da cultura o valor de ETc variou de 4,86 $\mathrm{mm} \mathrm{dia}^{-1}$ a 2,95 $\mathrm{mm} \mathrm{dia}{ }^{-1}$. Silva et al. (2015) verificaram, para a fase final uma ETc de 2,52 $\mathrm{mm} \mathrm{dia}^{-1}$, mas Bastos et al. (2012) encontraram ETc de 4,6 $\mathrm{mm} \mathrm{dia}^{-1}$. Todavia, estes últimos autores realizaram seu experimento em Parnaíba - PI, em que as diferentes variáveis climáticas podem justificar a maior demanda hídrica na fase final, em relação à deste experimento.

Nas simulações realizadas para as condições climáticas (temperatura) atuais, a necessidade hídrica total do ciclo da melancia foi de 248,9 mm. Silva et al. (2015) verificaram ao final do ciclo da mesma cultura, uma necessidade total de 222,2 mm. Já Ferreira (2010), avaliando a demanda hídrica da melancieira, em Alvorada do Gurguéia - PI, observou que a evapotranspiração total da cultura foi de $381 \mathrm{~mm}$.

Na Tabela 2 constam, a cada fase fenológica da cultura: o Kc oriundo de experimentos locais, a ETo, calculada pelo CROPWAT, porém com a utilização da variável climática: temperatura (cenário B2 otimista), prevista pelo $4^{\circ}$ Relatório do IPCC, para as próximas décadas, e ETc.

Tabela 2. Variáveis referentes à evapotranspiração da cultura da melancia - IPCC (B2): ETo (Evapotranspiração de Referência), Kc (Coeficiente de cultivo) e ETc (Evapotranspiração da cultura).

\begin{tabular}{cccc}
\hline Fase fenológica & ETo $\left(\mathrm{mm} \cdot \mathrm{dia}^{-1}\right)$ & Kc & ETc $\left(\mathrm{mm}_{\text {dia }}{ }^{-1}\right)$ \\
\hline Inicial & 4,8 & 0,25 & $1,19-1,32$ \\
Desenvolvimento & $4,80-5,03$ & $0,57-1,36$ & $1,32-6,57$ \\
Intermediaria & 5,03 & 1,36 & $6,57-5,10$ \\
Final & $5,03-4,65$ & 0,61 & $5,10-3,04$ \\
\hline
\end{tabular}


Na formulação da Tabela 3, o Coeficiente cultural (Kc) utilizado foi o mesmo da Tabela 2, mas com mudanças nos dados de temperatura, que constam no cenário
A2 (pessimista), o que, consequentemente, resultou em evapotranspirações de referência (ETo) e evapotranspirações da cultura (ETc) diferenciados.

Tabela 3. Variáveis referentes à evapotranspiração da cultura da melancia - IPCC (A2): ETo (Evapotranspiração de Referência), Kc (Coeficiente de cultivo) e ETc (Evapotranspiração da cultura).

\begin{tabular}{cccc}
\hline Fase fenológica & ETo $\left(\mathrm{mm} \cdot \mathrm{dia}^{-1}\right)$ & $\mathrm{Kc}$ & $\mathrm{ETc}\left(\mathrm{mm} \cdot \mathrm{dia}^{-1}\right)$ \\
\hline Inicial & 4,89 & 0,25 & $1,24-1,37$ \\
Desenvolvimento & $4,89-5,20$ & $0,57-1,36$ & $1,37-6,95$ \\
Intermediaria & 5,2 & 1,36 & $6,95-5,43$ \\
Final & $5,20-4,93$ & 0,61 & $5,43-3,30$ \\
\hline
\end{tabular}

Analisando a fase inicial do ciclo da cultura da melancia, para os cenários B2 e A2 (Tabelas 2 e 3), a ETc variou de 1,19 a 1,32 $\mathrm{mm} \mathrm{dia}^{-1}$ e de 1,24 a $1,37 \mathrm{~mm} \mathrm{dia}^{-1}$, respectivamente. É notório o aumento na demanda hídrica da cultura da melancia, quando há uma elevação na temperatura, pois aumenta-se a evapotranspiração de referência (ETo), e por consequência, a evapotranspiração da cultura (ETc).

Já na fase de desenvolvimento a ETc em B2 chegou a 6,57 $\mathrm{mm} \mathrm{dia}^{-1}$, enquanto nas condições do cenário A2 alcançou 6,95 mm $\mathrm{dia}^{-1}$. Carvalho et al. (2007) encontraram resultados semelhantes (ETc máxima média de 6,7 $\mathrm{mm} \mathrm{dia}^{-1}$ ), para as condições climáticas do litoral cearense. Verificou-se que a ETc calculada no cenário B2 (otimista), nas fases de maior demanda hídrica da melancia, subestimam as ETc's calculadas para o cenário A2 (pessimista), pois esse comportamento justifica-se, principalmente, pela ETo mais elevada, devido ao aumento da temperatura, nas condições do cenário A2. Ferreira et al. (2015) ao estudarem a demanda hídrica da melancia no Piauí, também verificaram maior necessidade hídrica da cultura na supracitada fase fenológica, com consumo médio de 5,2 mm.dia ${ }^{-1}$.
$\mathrm{Na}$ fase final do ciclo da cultura, a ETc de A2 (5,43 a 3,30 mm dia $\left.^{-1}\right)$ foi superior à ETc para B2 (5,10 a 3,04 mm dia $\left.{ }^{-1}\right)$. No caso da simulação para as condições climáticas do cenário B2 (otimista), a necessidade hídrica total do ciclo da melancia foi de 263,6 mm. Já quanto à simulação para o cenário A2 (pessimista), a necessidade hídrica total do ciclo da melancia foi de 278,7 $\mathrm{mm}$. Investigando as necessidades hídricas da melancia, no interior do Ceará, Souza et al. (2012) verificaram ao longo do ciclo da melancia irrigada uma demanda hídrica total de 265,8 $\mathrm{mm}$.

Segundo Vianna et al. (2012) as consequências das alterações climáticas são inexoráveis. Ademais, o Nordeste brasileiro é particularmente vulnerável, pois culturas que são tradicionalmente resistentes à seca vão alcançar seus limites de resistência tão logo a precipitação e temperatura atinjam os valores limites preconizados pelo cenário A2.

$\mathrm{Na}$ Figura 1 pode ser visto o comportamento comparativo da evapotranspiração da melancia, calculada com temperatura atual, com temperatura prevista pelo IPCC para o cenário B2, e com o aumento da temperatura previsto para A2. 


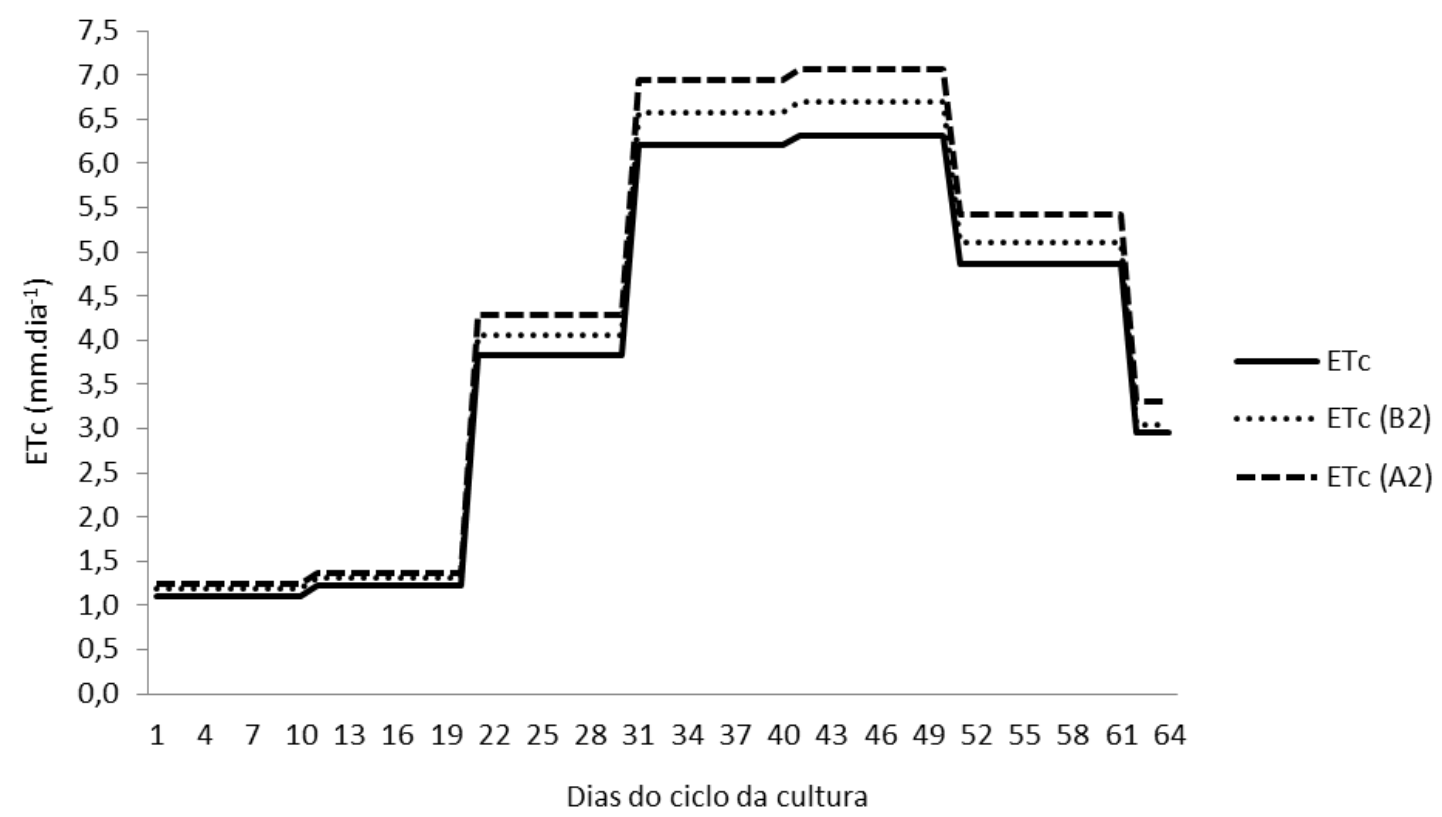

Figura 1. Evapotranspiração da melancia com ETc atual, ETc no cenário B2 e ETc no cenário A2.

Analisando a Figura 1, verificou-se que a ETc para o cenário A2 foi superior à B2, que por sua vez foi maior que a ETc calculada com dados climáticos atuais. Além do mais, vale salientar que os valores verificadores de ETc (cenários B2 e A2) foram superiores aos citados por Allen et al. (2006). Logicamente, os estudos dos referidos autores foram realizados para as condições climáticas do mediterrâneo; o que a princípio, gerariam demandas hídricas bem inferiores às verificadas em clima tropical.

Fato importante é que tanto para o cenário B2 como para o A2, as temperaturas máximas médias de Teresina alcançariam 37 ${ }^{\circ} \mathrm{C}$ a $39{ }^{\circ} \mathrm{C}$, não somente aumentando a necessidade hídrica da cultura da melancia, mas também podendo ocasionar problemas de adaptação da cultura no campo. Segundo Rezende et al. (2010) as cucurbitáceas se adaptam bem às zonas quentes e semiáridas, com alta luminosidade, mas com temperaturas do ar variando entre $18{ }^{\circ} \mathrm{C}$ a $30^{\circ} \mathrm{C}$.

Após a análise dos resultados é claramente visível que com o aumento da temperatura, previsto pelo IPCC, a demanda de água das culturas será aumentada, ou seja, para produzir a mesma quantidade de alimento, o produtor terá que irrigar, em termos médios, cerca de $(5,9 \%)$ a mais, no caso do cenário B2 e $(11,9 \%)$ a mais no caso do cenário A2. O cenário de aumento de temperatura é real e foi corroborado por Met Office (2010) que sugerem que, mesmo com a estabilização da temperatura, os efeitos do aquecimento global continuarão e serão mais drásticos na América do Sul, no Sul da África e na Austrália.

\section{CONCLUSÕES}

A pesquisa comprovou que, com o aumento da temperatura previsto pelo IPCC (cenários B2 e A2) para as próximas décadas, a demanda de água da cultura da melancia deverá se elevar, nas condições climáticas de Teresina - PI.

\section{REFERÊNCIAS}

ABREU, J. P. M.; PEREIRA, L. S. Impactos e vulnerabilidade da agricultura resultantes das alterações climáticas. p. 461-482. In: PEREIRA, L. S.; VICTORIA, F. R. B.; PAREDES, P.; GARCIA, M.; PALACIOS, E.; TORRECILLAS, A. (eds) 2010. Tecnologias para o Uso Sustentável da Água em Regadio. Edições Colibri e CEER, Lisboa Portugal. 2010. 
ALLEN, R.G.; PEREIRA, L.S.; RAES, D.; SMITH, J. Evapotranspiration del cultivo: guias para la determinación de los requerimientos de agua de los cultivos. Roma: FAO, 2006. 298 p. (Estudio Riego e Drenaje Paper, 56).

ALLEN, R. G.; PEREIRA, L. S.; RAES, D.; SMITH, M. Crop evapotranspiration: Guidelines for computing crop water requeriments. FAO Irrigation and Drainage. Paper 56. Rome, 300p. 1998.

ANNAN, C. Relatório anual da Organização das Nações Unidas - ONU. Roma, Itália. 2005. 256 p.

BASTOS, E. A.; SILVA, C. R.; RODRIGUES, B. H. N.; ANDRADE JUNIOR, A. S.; IBIAPINA, L. M. M. Evapotranspiration and crop coefficient of drip irrigated watermelon in Piauí coastline, Brazil. Revista Engenharia Agrícola, v.32, n.3, p.582-590, 2012.

CARVALHO, L. C. C. de, BEZERRA, F. M. L., CARVALHO, M. A. R. de. Evapotranspiração e coeficientes de cultivo da melancia sem sementes. Revista Ciência Agronômica, v.39, n.3, p.53-59. 2007.

DÍAZ, J. A., WEATHERHEAD, E. K., KNOX, J. W., CAMACHO, E. Climate change impacts on irrigation water requirements in the Guadalquivir river basin in Spain. Regional Environment Change, v.7, n.5, p.149-159. 2008.

FERREIRA, V. M.; KLAR, A. E.; ANDRADE JÚNIOR, A. S.; BASTOS, E. A.; OLIVEIRA, S. R. M. Evapotranspiração e coeficiente de cultura da melancia na microrregião de Teresina, PI, Brasil. Comunicata Scientiae, v.6, n.4, p.488-494, 2015.

FERREIRA, J. O. P. Evapotranspiração e coeficientes de cultura da melancieira irrigada por gotejamento em Alvorada do Gurguéia-PI. 2010. 121f. Tese (Doutorado em Agronomia-Produção Vegetal). Universidade Estadual "Júlio de Mesquita". Jaboticabal. 2010.

FIGUEIRÊDO, V. B.; MEDEIROS, J. F.; ZOCOLER, J. L.; SOBRINHO, J. E. Evapotranspiração da cultura da melancia irrigada com água de diferentes salinidades. Revista Engenharia Agrícola, v. 29, n. 2, p. 231-240, 2009.

IBGE. Sistema de recuperação automática Sidra: Produção agrícola Municipal: quantidade produzida, valor da produção, área plantada e área colhida da lavoura temporária. 2010. Disponível em: $<$ http://www.sidra.ibge.gov.br>. Acesso em 15 abr. 2016.

IPCC, Climate Change 2007: The Physical Science Basis. Contribution of Working Group I to the Fourth Assessment Report of the Intergovernmental Panel on Climate Change. Cambridge University Press, Cambridge, United Kingdom and New York, NY, USA, 996 p. 2007.

MARENGO, J. A. Vulnerabilidade, impactos e adaptação à mudança do clima no semi-árido do Brasil - Parcerias Estratégicas - n.27- Brasília, 2008, 36p.

MET OFFICE (2010). Cutting greenhouse gases will be no quick fix for our weather Adapting to Climate Change Issue 13. Disponível em: <www.metoffice.gov.uk> Acesso em 10 jul. 2016.

MINUZZI, R. B.; RIBEIRO, A. J.; SILVA, D. O.; KUNESKI, A. C. Estimativa da evapotranspiração de referência diária por penman-monteith fao com dados de temperatura do ar para Santa Catarina. Revista Irriga, v. 19, n. 4, p. 548-558, 2014.

MIRANDA, F. R. de; OLVEIRA, J. J. G.; SOUZA, F. Evapotranspiração máximas e coeficientes de cultivo para a cultura da melancia irrigada por gotejamento. Revista 
Ciência Agronômica, v. 35, n. 1, p. 36-46, 2004.

REZENDE, G. M. DE, DIAS, R. DE C. S., COSTA, N. D. CIMA. In: DIAS, R. DE C. S., REZENDE, G.M. DE, COSTA, N. D. Sistema de produção de melancia. 2010. Disponível em:<http://sistemasdeproducao.cnptia.embrapa .br/FontesHTML/Melancia/SistemaProducao Melancia/clima.htm>. Acesso em 20 jun. 2016.

SALES, C.; GERALDINI, F.; PAGLIUCA, L. Volume exportado de melancia é o maior da década. 2016. Disponível em: $<$ http://www.hfbrasil.org.br/exportacao-demelancia-1.aspx $>$. Acesso em: 20 de jun. de 2016.

SALES, C.; OLIVEIRA, C. A.; SPADETO, R. P. P.; POSSE, R. A. Requerimento de água para irrigação do milho na região São Mateus, ES. In: XXV CONIRD - Congresso Nacional de Irrigação e Drenagem 08 a 13 de novembro de 2015. Anais... UFS - São Cristóvão/SE. p. 99-104.

SANTOS, D. N.; SILVA, V. P. R.; SOUSA, F. A. S.; SILVA, R. A. Estudo de alguns cenários climáticos para o Nordeste do Brasil - Revista Brasileira de Engenharia Agrícola e Ambiental, v.14, n.5. p. 492-500, 2010.

SARAIVA, K. R.; SOUZA, F. de. O efeito das mudanças climáticas nas necessidades hídricas do feijão-caupi no curu-pentecoste utilizando o modelo "ISAREG". Revista Brasileira de Agricultura Irrigada, v. 6, n. 1, p. 8-13. 2013.
SARAIVA, K. R.; BEZERRA, F. M. L.; SOUZA, F. de.; CAMBOIM NETO, L. F. Aplicação do "ISAREG" no manejo da irrigação na cultura da melancia no Baixo Acaraú, Ceará. Revista Ciência Agronômica, v. 44, n. 1, p. 53-60, 2013.

SILVA, E. M. P.; ANDRADE JUNIOR, A. S.; BASTOS, E. A.; VIANA, T. V. A. Evapotranspiração e coeficiente da cultura da melancia em solo sob palhada e solo convencional. Revista Irriga, v. 20, n. 1, p. 154-164, 2015.

SILVA JÚNIOR, J. L. C.; COSTA, L. C.; AMORIM, M. C.; BARBOSA, F. J. Mudanças climáticas e agricultura: um estudo de casos para as culturas do milho e do feijão em Minas Gerais. Boletim da Sociedade Brasileira de Meteorologia. v.30, n.4. São Paulo. 28p. 2007.

SOUZA, M. M.; PEREIRA, K. A.; DUARTE, F. N. F. B.; XEREZ, P. C.; VIANA, R. S.; ARRUDA, J. M. Manejo de irrigação com base no clima na cultura da melancia no município de Iguatu-CE. In: Inovagri International Meeting 2012. Maio. Anais... Fortaleza, CE.

VIANNA, J. N. S.; PEREIRA, M. C.; DUARTE, L. M. G.; WEHRMANN, M. E. O papel das oleaginosas em um cenário de mudanças climáticas no semiárido brasileiro. Revista Brasileira de Geografia Física, v.6, n.3, p.1426-1445, 2012. 